\title{
WEB CONFERENCING SOFTWARE SELECTION WITH INTERVAL-VALUED FUZZY PARAMETERIZED INTUITIONISTIC FUZZY SOFT SETS
}

\author{
Esra Çakır \\ Department of Industrial Engineering, Galatasaray University, Istanbul, (Turkey). \\ E-mail: ecakir@gsu.edu.tr \\ ORCID: https://orcid.org/0000-0003-4134-7679
}

Ziya Ulukan

Department of Industrial Engineering, Galatasaray University, Istanbul, (Turkey).

E-mail:zulukan@gsu.edu.tr

ORCID: https://orcid.org/ 0000-0003-4805-2726

Recepción: 03/12/2020 Aceptación: 18/02/2021 Publicación: 07/05/2021

\section{Gitación sugerida:}

Çakır, E., y Ulukan, Z. (2021). Web Conferencing Software Selection with Interval-Valued Fuzzy Parameterized Intuitionistic Fuzzy Soft Sets. 3C Tecnología. Glosas de innovación aplicadas a la pyme, Edición Especial, (mayo 2021), 53-65. https://doi.org/10.17993/3ctecno.2021.specialissue7.53-65 


\section{ABSTRACT}

Since COVID-19 has become a pandemic, education has been interrupted in many countries, and training has been temporarily resumed on online platforms. But it is difficult to determine which of the many existing web (or video) conferencing software is more suitable for class education. The aim of this study is to sort these platforms according to the criteria determined by experts and select the best one among different alternatives by using interval-valued fuzzy parameterized intuitionistic fuzzy soft sets. In the application, seven experts determined the six criteria and their interval-valued fuzzy weights depending on educational needs. The decision makers are evaluated ten video conferencing tools for educational institutions by using interval-valued fuzzy parameterized intuitionistic fuzzy soft sets. Finally, using the proper operators, the alternatives are sorted and the best video conferencing tool for class education. The results are intended to guide future research.

\section{KEYWORDS}

Fuzzy MCDM, Interval-valued fuzzy sets, Intuitionistic fuzzy soft sets, Web conferencing tool, Software selection. 


\section{INTRODUCTION}

Due to the Covid-19 epidemic, countries had to take a break from education for a while and sought new ways to continue. After curfews came in many countries, educational institutions started looking for a suitable online video conferencing platform. These software allow participants to conduct or attend meetings via the internet. They enable remote meetings based on VoIP, online video, instant messaging, file sharing, and screen sharing. Online video conferencing is fundamental to many organizations conduct business in recent years. However, it has also become a must for formal education since the announcement of the pandemic.

Web conferencing software includes presentations or webinars, conference calls, video meetings with multiple participants, running product demos and training, one-on-one meetings with remote employees and face-to-face customer support. They are also useful in enhancing communications, reducing travel costs and increasing efficiency (Grant \& Cheon, 2007; Roehrs, 2013). By synchronous web conference communication, the gap between digital technologies and face-to-face teaching can be filled and this also encourages students to be attracted toward learning and in support of their self-working (Nedeva, Dineva \& Atanasov, 2014).

In order to adapt these platforms, which are very advantageous in the period when they are obliged to distance education, to their systems quickly and reliably, institutions should make decisions according to some criteria. Therefore, it is necessary to use expert opinions to select them and uncertainties views must also be considered. The fuzzy set proposed by Zadeh (1965) and improved by Atanassov (1986) as intuitionistic fuzzy sets are suitable for this application. Also, interval valued fuzzy sets (Atanassov \& Gargov, 1989) and intuitionistic fuzzy soft sets (Molodtsov, 1999) can sort alternatives and the robustness of their combined version has been proven (Nedeva et al., 2014). Jiang et al. (2010) proposed the notion of the interval-valued intuitionistic fuzzy soft set theory. It is an interval-valued fuzzy extension of the intuitionistic fuzzy soft set theory or an intuitionistic fuzzy extension of the interval-valued fuzzy soft set theory. The basic properties of the interval-valued intuitionistic fuzzy soft sets are also presented in their study. Deli and Karataş (2016) defined interval-valued intuitionistic fuzzy parameterized soft sets by combining the interval-valued 
intuitionistic fuzzy sets and the soft sets from parametrization point of view. By using soft level sets, they construct a parameter reduction method. Tripathy and Panigrahi (2016) extend hybrid model of interval valued fuzzy set and soft set to define interval valued intuitionistic fuzzy parameterized soft set (IVIFPSS) and establish their properties. They put forth two algorithms in decision-making. Aydın and Enginoğlu (2020) proposed the concept of interval-valued intuitionistic fuzzy parameterized interval-valued intuitionistic fuzzy soft sets (d-sets) and presents several of its basic properties. By using d-sets, they suggest a new soft decision-making method and apply it to a problem concerning the eligibility of candidates for two vacant positions in an online job advertisement.

The contribution of this study to the existing literature is to sort web conferencing platforms according to the criteria determined by experts and select the best one among ten options by using interval valued fuzzy parameterized intuitionistic fuzzy soft sets.

The rest of the article is organized as follows. Section 2 examines preliminaries and ranking methodology of interval valued fuzzy parameterized intuitionistic fuzzy soft sets. Section 3 presents numerical application in the selection of web conferencing tool. Conclusion of the study are stated in Section 4, along with future research.

\section{METHODOLOGY}

In this section, the preliminaries and definitions of the interval valued fuzzy parameterized intuitionistic fuzzy soft sets (IVFPIFS) and aggregation operator are introduced. The steps of methodology are given at the end of the section.

\subsection{PRELIMINARIES}

Definition 1. (Atanassov \& Gargov, 1989) Let $A$ be a collection of objects (points) denoted by $a_{1}$. Also, let $([0,1])$ be the set of all closed subintervals of the interval $[0,1]$. Then, an interval-valued intuitionistic fuzzy set $(I V I F S) Z$ in $A$ is defined as

$$
Z=\left\{\prec \gamma_{\mathrm{Z}}^{+}, \gamma_{\mathrm{Z}}^{-}>/ \mathrm{a}_{\mathrm{i}}: \mathrm{a}_{\mathrm{i}} \in A\right\}
$$

where $\gamma_{Z}^{+}=\left[\underline{\gamma_{Z}^{+}}\left(a_{i}\right), \overline{\gamma_{Z}^{+}}\left(a_{i}\right)\right], \gamma_{\bar{Z}}^{-}=\left[\underline{\gamma_{Z}^{-}}\left(a_{i}\right), \overline{\gamma_{Z}^{-}}\left(a_{i}\right)\right]: A \rightarrow \operatorname{Int}([0,1])$ are respectively called the membership function and the non-membership function of $Z$ with property $0 \leq \underline{\gamma_{Z}^{+}}\left(a_{i}\right)+$ 
$\underline{\gamma_{Z}^{-}}\left(a_{i}\right) \leq 1$ and $0 \leq \overline{\gamma_{Z}^{+}}\left(a_{i}\right)+\overline{\gamma_{Z}^{-}}\left(a_{i}\right) \leq 1$. The values $\underline{\gamma_{Z}^{+}}\left(a_{i}\right)$ and $\overline{\gamma_{Z}^{+}}\left(a_{i}\right)$ denote the lower and upper degrees of membership of the element $a_{i} \in A$, and also the values $\underline{\gamma_{\mathbf{Z}}^{-}}\left(\mathbf{a}_{\mathbf{i}}\right)$ and $\overline{\gamma_{\bar{Z}}}\left(a_{\mathbf{i}}\right)$ denote the lower and upper degrees of non-membership of the element $a_{i} \in A$, respectively. Note that $\gamma_{i}^{+}$and $\gamma_{i}^{-}$notations can be use instead of $\gamma_{\mathbf{Z}}^{+}\left(\mathbf{a}_{\mathbf{i}}\right)$ and $\gamma_{\mathbf{Z}}^{-}\left(\mathbf{a}_{\mathbf{i}}\right)$ for $\mathbf{a}_{\mathrm{i}} \in A$.

Definition 2. (Atanassov \& Gargov, 1989) Let $\gamma_{1}=\left\langle\left[\underline{\gamma_{1}^{+}}, \overline{\gamma_{1}^{+}}\right],\left[\underline{\gamma_{1}^{-}}, \overline{\gamma_{1}^{-}}\right]\right\rangle$and $\gamma_{2}=<\left[\underline{\gamma_{2}^{+}}, \overline{\gamma_{2}^{+}}\right],\left[\underline{\gamma_{2}^{-}}, \overline{\gamma_{2}^{-}}\right]>$be two IVIF values. Then, the operations $\oplus$ and $\otimes$ for $\gamma_{1}$ and $\gamma_{2}$ are respectively defined as follows:

$$
\begin{aligned}
& \gamma_{1} \oplus \gamma_{2}=<\left[\underline{\gamma_{1}^{+}}+\underline{\gamma_{2}^{+}}-\underline{\gamma_{1}^{+}} \cdot \underline{\gamma_{2}^{+}}, \overline{\gamma_{1}^{+}}+\overline{\gamma_{2}^{+}}-\overline{\gamma_{1}^{+}} \cdot \overline{\gamma_{2}^{+}}\right],\left[\underline{\gamma_{1}^{-}} \cdot \underline{\gamma_{2}^{-}}, \overline{\gamma_{1}^{-}} \cdot \overline{\gamma_{2}^{-}}\right]> \\
& \gamma_{1} \otimes \gamma_{2}=<\left[\underline{\gamma_{1}^{+}} \cdot \underline{\gamma_{2}^{+}}, \overline{\gamma_{1}^{+}} \cdot \overline{\gamma_{2}^{+}}\right],\left[\underline{\gamma_{1}^{-}}+\underline{\gamma_{2}^{-}}-\underline{\gamma_{1}^{-}} \cdot \underline{\gamma_{2}^{-}}, \overline{\gamma_{1}^{-}}+\overline{\gamma_{2}^{-}}-\overline{\gamma_{1}^{-}} \cdot \overline{\gamma_{2}^{-}}\right]>
\end{aligned}
$$

Definition 3. (Tan, 2011; Xu, 2010) Let $\gamma_{\mathrm{i}}=<\gamma_{\mathrm{i}}^{+}, \gamma_{\mathrm{i}}^{-}>=<\left[\underline{\gamma_{i}^{+}}, \overline{\gamma_{i}^{+}}\right],\left[\underline{\gamma_{i}^{-}}, \overline{\gamma_{i}^{-}}\right]>$be the IVIF value of $a_{i} \in A$. Then, the score function and accuracy function of $\gamma_{1}$ are respectively defined as follows:

$$
\begin{aligned}
& \left.\mathrm{s}\left(\gamma_{\mathrm{i}}\right)=\frac{1}{2} \underline{\gamma_{i}^{+}}-\underline{\gamma_{i}^{-}}+\overline{\gamma_{i}^{+}}-\overline{\gamma_{i}^{-}}\right) \\
& \left.\mathrm{a}\left(\gamma_{\mathrm{i}}\right)=\frac{1}{2} \underline{\gamma_{i}^{+}}+\underline{\gamma_{i}^{-}}+\overline{\gamma_{i}^{+}}+\overline{\gamma_{i}^{-}}\right)
\end{aligned}
$$

To compare two IVIF values $\gamma_{1}$ and $\gamma_{2}$, a ranking method is defined as follows:

$$
\begin{aligned}
& \text { If } \mathrm{s}\left(\gamma_{1}\right)<\mathrm{s}\left(\gamma_{2}\right) \text { then } \gamma_{1}<\gamma_{2}, \\
& \begin{aligned}
\text { If } \mathrm{s}\left(\gamma_{1}\right) & =\mathrm{s}\left(\gamma_{2}\right) \text { then } \\
& \text { if } \mathrm{a}\left(\gamma_{1}\right)<\mathrm{a}\left(\gamma_{2}\right) \text { then } \gamma_{1}<\gamma_{2} . \\
& \text { if } \mathrm{a}\left(\gamma_{1}\right)=\mathrm{a}\left(\gamma_{2}\right) \text { then } \gamma_{1}=\gamma_{2} .
\end{aligned}
\end{aligned}
$$

Definition 4. Let $U$ be a universal set and $\mathrm{C}$ be the set of parameters. Also, let $\mathrm{I}$ be an interval-valued fuzzy set over $\mathrm{C}$ with the membership function $\omega_{1}: \mathrm{C} \rightarrow \operatorname{Int}([0,1])$. Then, an interval-valued fuzzy parameterized intuitionistic fuzzy soft set (IVFPIFS set) $\Psi_{\mathrm{X}}$ on $U$ is a set of ordered pairs 


$$
\Psi_{\mathrm{I}}=\left\{\left(\omega_{\mathrm{I}}\left(c_{\mathrm{j}}\right) / c_{\mathrm{j}}, \Psi_{\mathrm{I}}\left(c_{\mathrm{j}}\right)\right): c_{\mathrm{j}} \in \mathrm{C}, \Psi_{\mathrm{I}}\left(c_{\mathrm{j}}\right) \in I F S(U)\right\}
$$

where the function $\Psi_{\mathrm{I}}: \mathrm{G} \rightarrow \operatorname{IFS}(U)$ such that $\Psi_{\mathrm{I}}\left(c_{\mathrm{j}}\right)=\emptyset$ if $\omega_{\mathrm{I}}\left(c_{\mathrm{j}}\right)=$ $\left[\underline{\omega_{\mathrm{I}}}\left(c_{\mathrm{j}}\right), \overline{\omega_{\mathrm{I}}}\left(c_{\mathrm{j}}\right)\right]=[0,0]$. Note that $\operatorname{IVFPIF}(U)$ notation denotes the set of all intervalvalued fuzzy parameterized intuitionistic fuzzy soft sets on $U$.

Definition 5. Let $\Psi_{\mathrm{I}}=\left\{\left(\omega_{\mathrm{j}} / c_{\mathrm{j}},\left\{\prec \gamma_{\mathrm{ij}}^{+}, \gamma_{\mathrm{ij}}^{-}>/ a_{\mathrm{i}}: a_{\mathrm{i}} \in U\right\}\right): c_{\mathrm{j}} \in \mathrm{C}\right.$ be an IVFPIFS set,where $\gamma_{\mathrm{ij}}=\left\langle\gamma_{\mathrm{ij}}^{+}, \gamma_{\mathrm{ij}}^{-}>i=1,2, \ldots, n\right.$ indicates an intuitionistic fuzzy value when the alternative $a_{1}$ is assessed with respect to the parameter $c_{\mathrm{j}}$ and $\omega_{\mathbf{I}}\left(c_{\mathbf{j}}\right)=\omega_{\mathrm{j}}=\left[\omega_{\mathrm{j}}, \overline{\omega_{\mathrm{j}}}\right]$ indicates an interval-valued fuzzy value of the parameter $c_{\mathrm{j}}$. Then,

- the first IVFPIFS-aggregation operator, denoted by IVFPIFS $S_{\text {aggr }}^{1}$, is defined by

$$
\operatorname{IVFPIFS}_{\text {aggr }}^{1}:(\mathrm{C}) \times \operatorname{IVFPIFSS}(U) \rightarrow \operatorname{IVIFS}(U), \operatorname{IVFPIFS}_{\text {aggr }}^{1}\left(\mathrm{I}, \dot{\Psi}_{\mathrm{I}}\right)=\dot{\Psi}_{\mathrm{I}}
$$

where $\dot{\Psi}_{\mathrm{I}}=\left\{\left\langle\left[\underline{\dot{\gamma}_{i}^{+}}, \overline{\dot{\gamma}_{i}^{+}}\right],\left[\underline{\dot{\gamma}_{i}^{-}}, \overline{\dot{\gamma}_{i}^{-}}\right]>/ a_{\mathrm{i}}: a_{\mathrm{i}} \in U\right\}\right.$ which is an interval-valued intuitionistic fuzzy set on $U$. The membership degree $\dot{\gamma}_{i}^{+}=\left[\underline{\dot{\gamma}_{i}^{+}}, \overline{\hat{\gamma}_{i}^{+}}\right]$and the non-membership degree $\dot{\gamma}_{i}^{-}=\left[\underline{\hat{\gamma}_{i}^{-}}, \overline{\hat{\gamma}_{i}^{-}}\right]$of $a_{i} \in U$ is defined as follows:

$$
\begin{gathered}
\dot{\gamma}_{\mathrm{i}}^{+}=\left[1-\prod_{j=1}^{m}\left(1-\underline{\omega_{\mathrm{j}}} \cdot \gamma_{\mathrm{ij}}^{+}\right), 1-\prod_{j=1}^{m}\left(1-\overline{\omega_{\mathrm{j}}} \cdot \gamma_{\mathrm{ij}}^{+}\right)\right] \\
\dot{\gamma}_{\mathrm{i}}^{-}=\left[\prod_{j=1}^{m} \underline{\omega_{\mathrm{j}}} \cdot \gamma_{\mathrm{ij}}^{-}, \prod_{j=1}^{m} \overline{\omega_{\mathrm{j}}} \cdot \gamma_{\mathrm{ij}}^{-}\right]
\end{gathered}
$$

where $m$ denotes the number of parameters in C.

- the second IVFPIFS-aggregation operator, denoted by IVFPIFS 1 aggr, is defined by

$$
\operatorname{IVFPIFS}_{\text {aggr }}^{2}:(\mathrm{C}) \times \operatorname{IVFPIFSS}(U) \rightarrow \operatorname{IVIFS}(U), \operatorname{IVFPIFS} \mathrm{aggr}^{2}\left(\mathrm{I}, \dot{\Psi}_{\mathrm{I}}\right)=\ddot{\Psi}_{\mathrm{I}}
$$

where $\ddot{\Psi}_{\mathrm{I}}=\left\{<\left[\underline{\ddot{\gamma}_{i}^{+}}, \overline{\ddot{\gamma}_{\mathrm{i}}^{+}}\right],\left[\underline{\ddot{\gamma}_{i}^{-}}, \overline{\ddot{\gamma}_{\mathrm{i}}^{-}}\right]>/ a_{\mathrm{i}}: a_{\mathrm{i}} \in U\right\}$ which is an interval-valued intuitionistic fuzzy set on $U$. The membership degree $\ddot{\gamma}_{i}^{+}=\left[\underline{\ddot{\gamma}_{i}^{+}}, \overline{\ddot{\gamma}_{i}^{+}}\right]$and the non-membership degree $\left.\ddot{\gamma}_{\mathrm{i}}^{-}=\underline{\ddot{\gamma}_{\mathrm{i}}^{-}}, \overline{\ddot{\gamma}_{\mathrm{i}}^{-}}\right]$of $a_{\mathrm{i}} \in U$ is defined as follows:

$$
\begin{gathered}
\ddot{\gamma}_{\mathrm{i}}^{+}=\left[\prod_{j=1}^{m} \underline{\omega_{\mathrm{j}}} \cdot \gamma_{\mathrm{ij}}^{+}, \prod_{j=1}^{m} \overline{\omega_{\mathrm{j}}} \cdot \gamma_{\mathrm{ij}}^{+}\right] \\
\ddot{\gamma}_{\mathrm{i}}^{-}=\left[1-\prod_{j=1}^{m}\left(1-\underline{\omega_{\mathrm{j}}} \cdot \gamma_{\mathrm{ij}}^{-}\right), 1-\prod_{j=1}^{m}\left(1-\overline{\omega_{\mathrm{j}}} \cdot \gamma_{\mathrm{ij}}^{-}\right)\right]
\end{gathered}
$$

where $m$ denotes the number of parameters in C. 


\subsection{ALGORITHM}

This methodology is stated for multi criteria decision making problem. It uses interval valued fuzzy parameterized intuitionistic fuzzy soft sets to express decision makers opinion and generate interval valued intuitionistic fuzzy sets by using two different aggregation operators (Nedeva et al., 2014). Score and accuracy functions help to sort the alternatives and to select best option. The steps of this methodology are as follows:

- Step 1: Set the problem. $a_{1}$ indicates alternative $i$.

- Step 2: Select experts and determine criteria with weights by experts. $\mathrm{C}_{\mathrm{j}}$ indicates criterion $\mathrm{j}$ and its interval-valued fuzzy weight is expressed as $\omega_{j}=\left[\underline{\omega_{j}}, \overline{\omega_{j}}\right]$.

- Step 3: Collect the fuzzy decision matrices with IVFPIFS set by experts and aggregate them by fuzzy geometric mean method of Buckley (Buckley, 1985).

- Step 4: Obtain the first and second aggregate interval-valued intuitionistic fuzzy set $\left\langle\dot{\gamma}_{i}^{+}, \dot{\gamma}_{i}^{-}>\right.$and $\prec \ddot{\gamma}_{i}^{+}, \ddot{\gamma}_{i}^{-}>$of alternatives by using IVFPIFS-aggregation operators given in Def. 5 .

- Step 5: Calculate the IVIF values $\left.\dddot{\gamma}_{i}=<\dddot{\gamma}_{i}^{+}, \dddot{\gamma}_{i}^{-}\right\rangle$of alternatives by applying $\oplus$ operation given in Def. 2.

- Step 6: By using the score function and accuracy function on IVIF values given in Def. 3, compare the alternatives and set the highest score alternative as the best option.

\section{APPLICATION}

After being declared COVID-19 pandemic, educational institutions in many countries took a break for a while. In order not to fall behind in education, many institutions have evaluated opportunities for distance education. With the web (or video) conferencing software, teachers had the chance to teach their classes during the periods they set. However, these systems had to be selected and adapted to the institutions in a very short time. So, it is a problem to determine which service is better. In this application, the alternatives are evaluated, and the criteria are determined with the help of experts in Turkey. Platforms are ranked using interval valued fuzzy parameterized intuitionistic fuzzy soft sets, and the alternative with 
the highest score is evaluated as the best platform. According to the previous section, this application is implemented step by step as follows:

- Step 1: Web conferencing software used in Turkey selection is set as the problem. The ten most frequently used software during the pandemic period are determined as alternatives. The alternatives are presented as $\mathrm{A}=\left\{a_{1}, a_{2}, a_{3}, a_{4}, a_{5}, a_{6}, a_{7}, a_{8}, a_{9}\right.$, $\left.a_{10}\right\}$.

- Step 2: Seven experts are selected, and they determined the criteria depending on educational needs and the features of tools. These criteria are given with their interval-valued fuzzy weights in Table 1.

Table 1. The criteria for web conferencing software selection with their interval-valued fuzzy weights.

\begin{tabular}{|c|c|c|}
\hline Criteria code & Criteria & Interval-valued fuzzy weights " $\boldsymbol{\omega}_{\mathrm{i}}$ " \\
\hline $\boldsymbol{c}_{\boldsymbol{1}}$ & Performance and Compatibility & {$[0.6,0.9]$} \\
\hline $\boldsymbol{c}_{2}$ & File and Screen Sharing & {$[0.3,0.5]$} \\
\hline $\boldsymbol{c}_{3}$ & Online Meeting Quality and Recording & {$[0.6,0.8]$} \\
\hline $\boldsymbol{c}_{\boldsymbol{4}}$ & Implementation & {$[0.4,0.7]$} \\
\hline $\boldsymbol{c}_{5}$ & Security & {$[0.7,0.9]$} \\
\hline $\boldsymbol{c}_{\boldsymbol{6}}$ & Support System & {$[0.5,0.8]$} \\
\hline
\end{tabular}

Source: own elaboration.

- Step 3: Experts decided on the degree of membership of alternatives to specified criteria with IVFPIFS set expressions and their decision matrices are aggregated by fuzzy geometric mean method of Buckley. The aggregated decision matrix is represented in Table 2.

Table 2. The aggregated IVFPIFS decision matrix.

\begin{tabular}{|c|c|c|c|c|c|c|}
\hline Crt. & $c_{1}$ & $c_{2}$ & $c_{3}$ & $\mathrm{C}_{4}$ & $c_{5}$ & $c_{6}$ \\
\hline Alt. & $\omega_{1}=[0.6,0.9]$ & $\omega_{2}=[0.3,0.5]$ & $\omega_{3}=[0.6,0.8]$ & $\omega_{4}=[0.4,0.7]$ & $\omega_{5}=[0.7,0.9]$ & $\omega_{6}=[0.5,0.8]$ \\
\hline$a_{1}$ & $<0.72,0.22>$ & $<0.68,0.21>$ & $<0.86,0.12>$ & $<0.67,0.22>$ & $<0.67,0.21>$ & $<0.63,0.36>$ \\
\hline$a_{2}$ & $<0.71,0.19>$ & $<0.66,0.33>$ & $<0.83,0.11>$ & $<0.32,0.51>$ & $<0.63,0.18>$ & $<0.51,0.48>$ \\
\hline$a_{3}$ & $<0.63,0.31>$ & $<0.62,0.15>$ & $<0.73,0.23>$ & $<0.51,0.38>$ & $<0.65,0.25>$ & $<0.49,0.51>$ \\
\hline$a_{4}$ & $<0.65,0.33>$ & $<0.6,0.38>$ & $<0.82,0.1>$ & $<0.59,0.24>$ & $<0.6,0.22>$ & $<0.48,0.4>$ \\
\hline$a_{5}$ & $<0.68,0.29>$ & $<0.63,0.27>$ & $<0.82,0.05>$ & $<0.65,0.22>$ & $<0.63,0.2>$ & $<0.54,0.37>$ \\
\hline$a_{6}$ & $<0.65,0.23>$ & $<0.67,0.33>$ & $<0.78,0.22>$ & $<0.49,0.36>$ & $<0.63,0.14>$ & $<0.4,0.55>$ \\
\hline
\end{tabular}




\begin{tabular}{|c|c|c|c|c|c|c|}
\hline $\boldsymbol{a}_{\boldsymbol{7}}$ & $<0.68,0.17>$ & $<0.62,0.3>$ & $<0.77,0.09>$ & $<0.62,0.23>$ & $<0.59,0.31>$ & $<0.55,0.42>$ \\
\hline $\boldsymbol{a}_{\boldsymbol{g}}$ & $<0.66,0.24>$ & $<0.63,0.29>$ & $<0.73,0.14>$ & $<0.66,0.21>$ & $<0.65,0.24>$ & $<0.55,0.36>$ \\
\hline $\boldsymbol{a}_{\boldsymbol{g}}$ & $<0.59,0.4>$ & $<0.45,0.46>$ & $<0.69,0.15>$ & $<0.52,0.31>$ & $<0.54,0.28>$ & $<0.34,0.58>$ \\
\hline $\boldsymbol{a}_{10}$ & $<0.62,0.26>$ & $<0.56,0.41>$ & $<0.74,0.22>$ & $<0.48,0.36>$ & $<0.5,0.31>$ & $<0.63,0.33>$ \\
\hline
\end{tabular}

Source: own elaboration.

- Step 4: The first and second aggregate interval-valued intuitionistic fuzzy set $\prec \dot{\gamma}_{i}^{+}, \dot{\gamma}_{i}^{-}>$and $\prec \ddot{\gamma}_{i}^{+}, \ddot{\gamma}_{i}^{-}>$of alternatives by using IVFPIFS-aggregation operators are obtained as in Table 3 .

Table 3. The aggregate interval-valued intuitionistic fuzzy sets of alternatives.

\begin{tabular}{|c|c|c|}
\hline Alt. & $\prec \dot{\gamma}_{i}^{+}, \dot{\gamma}_{i}^{-} \succ=\prec\left[\underline{\dot{\gamma}_{i}^{+}}, \overline{\dot{\gamma}_{i}^{+}}\right],\left[\underline{\dot{\gamma}_{i}^{-}}, \overline{\dot{\gamma}_{i}^{-}}\right] \succ$ & $\prec \ddot{\gamma}_{i}^{+}, \ddot{\gamma}_{i}^{-} \succ=\prec\left[\underline{\ddot{\gamma}_{i}^{+}}, \overline{\ddot{\gamma}_{i}^{+}}\right],\left[\underline{\ddot{\gamma}_{i}^{-}}, \overline{\ddot{\gamma}_{i}^{-}}\right] \succ$ \\
\hline$a_{1}$ & $<[0.94173,0.99242],[0.0000013,0.0000167]>$ & $<[0.0018,0.021605],[0.518534,0.683016]>$ \\
\hline$a_{2}$ & $<[0.91607,0.98383],[0.0000045,0.0000551]>$ & $<[0.000604,0.007255],[0.605775,0.79045]>$ \\
\hline$a_{3}$ & $<[0.906801,0.97983],[0.0000078,0.000094]>$ & $<[0.0007003,0.008403],[0.65074,0.81673]>$ \\
\hline$a_{4}$ & $<[0.914425,0.983378],[0.000004,0.000048]>$ & $<[0.000821,0.009859],[0.59133,0.762296]>$ \\
\hline$a_{5}$ & $<[0.92635,0.98774],[0.0000009,0.0000115]>$ & $<[0.001174,0.014094],[0.52932,0.700298]>$ \\
\hline$a_{6}$ & $<[0.90677,0.97992],[0.0000069,0.0000839]>$ & $<[0.000634,0.007610],[0.62262,0.800249]>$ \\
\hline$a_{7}$ & $<[0.917029,0.98471],[0.000002,0.0000249]>$ & $<[0.000987,0.01185],[0.565806,0.731641]>$ \\
\hline$a_{g}$ & $<[0.91994,0.98553],[0.0000026,0.0000320]>$ & $<[0.001082,0.012994],[0.55262,0.716573]>$ \\
\hline$a_{g}$ & $<[0.86611,0.961247],[0.000021,0.0002521]>$ & $<[0.000264,0.003173],[0.70188,0.863861]>$ \\
\hline$a_{10}$ & $<[0.895486,0.97648],[0.000013,0.0001567]>$ & $<[0.000587,0.00704],[0.640429,0.800823]>$ \\
\hline
\end{tabular}

Source: own elaboration.

- Step 5: The IVIF values $\dddot{\gamma}_{i}=<\dddot{\gamma}_{i}^{+}, \dddot{\gamma}_{i}^{-}>$of alternatives by applying $\bigoplus$ operation is calculated as in Table 4.

- Step 6: The scores and accuracy values of IVIF values are presented in Table 5.

Table 4. The interval valued intuitionistic fuzzy values of alternatives.

\begin{tabular}{|c|c|}
\hline Alt. & $\dddot{\gamma}_{i}=\prec \dddot{\gamma}_{i}^{+}, \dddot{\gamma}_{i}^{-} \succ=\prec \dot{\gamma}_{i}^{+}, \dot{\gamma}_{i}^{-} \succ \oplus \prec \ddot{\gamma}_{i}^{+}, \ddot{\gamma}_{i}^{-} \succ$ \\
\hline $\boldsymbol{a}_{1}$ & $<[0.94184,0.992584],[0.000000722,0.0000114]>$ \\
\hline $\boldsymbol{a}_{2}$ & $<[0.916128,0.983951],[0.00000278,784538,0.0000435]>$ \\
\hline $\boldsymbol{a}_{3}$ & $<[0.906867,0.9800047],[0.00000509,652078,0.0000767]>$ \\
$\boldsymbol{a}_{4}$ & $<[0.914496,0.983541],[0.00000236,637966,0.0000366]>$
\end{tabular}




\section{Source: own elaboration.}

$<[0.926436,0.987917],[0.00000051,809846133,180987]>$

$<[0.906831,0.980079],[0.000004357,035645,0.0000672]>$

$<[0.917111,0.984898],[0.00000117,284747,0.0000182]>$

$<[0.920028,0.985722],[0.00000147,515918,0.0000229]>$

$<[0.866147,0.96137008],[0.0000147,0.0002177]>$

$<[0.8955477,0.9766463],[0.00000836,714534,0.0001254]>$

Table 5. The scores of alternatives.

Source: own elaboration.

\begin{tabular}{|c|c|c|}
\hline Alternative & Score $\mathbf{s}\left(\boldsymbol{a}_{\boldsymbol{1}}\right)$ & Accuracy $\mathbf{a}\left(\boldsymbol{a}_{\boldsymbol{1}}\right)$ \\
\hline $\boldsymbol{a}_{\boldsymbol{1}}$ & -0.02538 & 0.967219 \\
\hline $\boldsymbol{a}_{2}$ & -0.03393 & 0.950064 \\
\hline $\boldsymbol{a}_{\boldsymbol{3}}$ & -0.0366 & 0.943477 \\
\hline $\boldsymbol{a}_{\boldsymbol{4}}$ & -0.03454 & 0.949039 \\
\hline $\boldsymbol{a}_{5}$ & -0.03074 & 0.957181 \\
\hline $\boldsymbol{a}_{\boldsymbol{6}}$ & -0.03666 & 0.943491 \\
\hline $\boldsymbol{a}_{\boldsymbol{7}}$ & -0.0339 & 0.951015 \\
\hline $\boldsymbol{a}_{\boldsymbol{g}}$ & -0.03286 & 0.952888 \\
\hline $\boldsymbol{a}_{\boldsymbol{g}}$ & -0.04771 & 0.913875 \\
\hline $\boldsymbol{a}_{\boldsymbol{1 0}}$ & -0.04061 & 0.936164 \\
\hline
\end{tabular}

According to the scores, the ranking of the alternatives is $a_{1}>a_{5}>a_{8}>a_{7}>a_{2}>a_{4}>a_{3}>a_{6}>a_{10}$ $>a_{9}$. Since $a_{1}$ has the highest score, it is selected as the best option for education institutions based on determined criteria and expert's opinions.

\section{CONCLUSIONS}

Due to the COVID-19 outbreak, interest in web conferencing platforms has increased in educational institutions around the world. Since there are many different options, which software institutions choose is an important issue. The aim of this study is to compare ten video conferencing software according to the criteria determined by the experts with their imprecise opinions and to choose the best option by using interval valued fuzzy parameterized intuitionistic fuzzy soft sets. As a result of the study, alternatives are listed as 
$a_{1}>a_{5}>a_{8}>a_{7}>a_{2}>a_{4}>a_{3}>a_{6}>a_{10}>a_{9}$ and " $a_{1}$ " platform is chosen as the best video conferencing tool for educational institutions according to the determined criteria.

\section{ACKNOWLEDGEMENTS}

This work has been supported by the Scientific Research Projects Commission of Galatasaray University under grant number \# FBA-2020-1036.

\section{REFERENCES}

Atanassov, K. T. (1986) Intuitionistic fuzzy sets. Fuzzy Sets and Systems, 20(1), 87-96. https://doi.org/10.1016/S0165-0114(86)80034-3

Atanassov, K., \& Gargov, G. (1989). Interval-valued intuitionistic fuzzy sets. Fuzzy Sets and Systems, 31(3), 343-349. https://doi.org/10.1016/0165-0114(89)90205-4

Aydin, T., \& Enginoglu, S. (2020). Interval-valued intuitionistic fuzzy parameterized interval-valued intuitionistic fuzzy soft sets and their application in decision making. Journal of Ambient Intelligence and Humanized Computing, 12, 1541-1558. https://doi. org/10.1007/s 12652-020-02227-0

Buckley, J. J. (1985). Fuzzy hierarchical analysis. Fuzzy sets and Systems, 17(3), 233-247. https://doi.org/10.1016/0165-01 14(85)90090-9

Deli, I., \& Karataş, S. (2016). Interval Valued Intuitionistic Fuzzy Parameterized Soft Set Theory and Its Decision Making. Journal of Intelligent $\mathcal{E}^{2}$ Fuzzy Systems, 30(4), 20732082.https://www.researchgate.net/publication/298735300_Interval_valued_ intuitionistic_fuzzy_parameterized_soft_set_theory_and_its_decision_making

Grant,M.M.,\&Cheon,J.(2007). Thevalue of usingsynchronousconferencingforinstruction and students. Fournal of Interactive Online Learning, 6(3), 211-226. https: / / citeseerx.ist. psu.edu/viewdoc/download?doi=10.1.1.530.1145\&rep=rep 1\&type=pdf

Jiang, Y., Tang, Y. Ghen, Q., Liu, H., \& Tang, J. (2010). Interval-valued intuitionistic fuzzy soft sets and their properties. Computers and Mathematics with Applications, 60(3), 906-918. https://doi.org/10.1016/j.camwa.2010.05.036 
Molodtsov, D. (1999). Soft set theory-first results. Computers E्E Mathematics with Applications, 37(4-5), 19-31. https://doi.org/10.1016/S0898-1221(99)00056-5

Nedeva, V., Dineva, S., \& Atanasov, S. (2014). Effective E-learning Course With Web Conferencing. In V-th National Conference of E-Learning, Ruse, Bulgaria. https://doi. org/10.13140/2.1.2605.6648

Roehrs, B. (2013). Providing online classes with big bluebutton 2013-09-30a. http://www. slideshare.net/roehrsb/providing-on-line-classes-ith-big-bluebutton-20130930a

Tan, C. (2011). A multi-criteria interval-valued intuitionistic fuzzy group decision making with Choquet integral-based TOPSIS. Expert Systems with Applications, 38(4), 30233033. https://doi.org/10.1016/j.eswa.2010.08.092

Tripathy, B. K., \& Panigrahi, A. (2016). Interval-valued intuitionistic fuzzy parameterized soft set theory and its application in decision-making. In 10th International Conference on Intelligent Systems and Control (ISCO), 1-6. https://doi.org/10.1109/ ISCO.2016.7726952

Xu, Z. (2010). Choquet integrals of weighted intuitionistic fuzzy information. Information Sciences, 180(5), 726-736. https://doi.org/10.1016/j.ins.2009.11.011

Zadeh, L. A. (1965). Fuzzy sets. Information and Control, 8(3), 338-353. https://doi. org/10.1016/S0019-9958(65)90241-X 
